Arq. Bras. Med. Vet. Zootec., v.67, n.4, p.1119-1124, 2015

\title{
Estimativas de parâmetros genéticos para características de crescimento em ovinos da raça Suffolk no Brasil
}

\author{
[Estimates of genetic parameters for growth traits in Suffolk sheep in Brazil] \\ M.P. Pires, M.M. Farah ${ }^{1}$, L.O.D. Carreño ${ }^{1}$, A.T.H. Utsunomiya ${ }^{1}$, R.K. Ono ${ }^{1}$, \\ T.S. Bertipaglia ${ }^{1}$, R. Fonseca ${ }^{2}$ \\ ${ }^{1}$ Universidade Estadual Paulista "Júlio de Mesquita Filho"- Jaboticabal, SP \\ ${ }^{2}$ Universidade Estadual Paulista "Júlio de Mesquita Filho" - Dracena, SP
}

\begin{abstract}
RESUMO
O objetivo do trabalho foi estimar os parâmetros genéticos das características de crescimento da raça Suffolk, a fim de fornecer subsídio para a definição de estratégias de seleção para programas de melhoramento genético. Os dados analisados, coletados entre os anos de 2007 a 2009, são referentes a ovinos da raça Suffolk oriundos de uma propriedade localizada no Estado de São Paulo, Brasil, participante do programa de melhoramento genético Ovigol, conduzido pela empresa Aries Reprodução e Melhoramento Genético Ovino Ltda. em parceria com a empresa AbacusBio Limited, da Nova Zelândia. As características avaliadas foram: peso ao nascer (PN), ganho de peso pré-desmame (GPP) e peso ao desmame (PD), com número de registro de 1.039, 636 e 649 animais, respectivamente. Para análise estatística, utilizou-se o procedimento GLM do programa computacional SAS; para a consistência do pedigree e para as estimativas dos parâmetros genéticos, os programas computacionais utilizados foram Relax2: pedigree analysis program e o WOMBAT, respectivamente. O modelo linear geral incluiu sexo, grupo de contemporâneos (tipo de parto e ano de nascimento), covariável idade ao desmame (GPP e PD) com efeito quadrático, efeito genético aditivo direto, efeito de ambiente permanente materno e efeito residual. As herdabilidades para PN, GPP e PD foram 0,06 , 0,42 e 0,37 , respectivamente. As correlações genéticas entre PN e GPP, PN e PD e GPP e PD foram - $0,10,-0,03$ e 0,97 , respectivamente. Na população estudada, as características GPP e PD, apresentam altas respostas à seleção, ao contrário do PN. As características PN e GPP são suficientes para compor um índice de seleção, sendo aconselhável o monitoramento do PN na fase inicial do programa.
\end{abstract}

Palavras-chave: herdabilidade, melhoramento genético, ovinocultura, genética

\begin{abstract}
The aim of this study was to estimate genetic parameters for growth of Suffolk, in order to provide a basis for the definition of selection strategies for breeding programs. The data analyzed are for Suffolk sheep, collected between years 2007 to 2009, from a property located in the State of São Paulo - Brazil, a participant in the Ovigol breeding program conducted by the company (Aries Reproduction and Breeding Sheep Ltd.) in partnership with (AbacusBio) New Zealand Limited. The evaluated characteristics were: birth weight (PN), weight gain pre-weaning (GPP) and weaning weight (PD), with registration number 1039, 636 and 649 respectively. For statistical analysis we used the GLM procedure of SAS software, for the consistency of pedigree and genetic parameter estimates software Relax2: pedigree analysis program and WOMBAT, respectively, were used. The general linear model included sex, contemporary group (type of birth and year of birth), covariate age at weaning (GPP and PD) with a quadratic effect, direct genetic effect, maternal permanent environmental effect and residual effect. Heritability estimates for PN, PD and GPP were 0.06, 0.42 and 0.37, respectively. Genetic correlations between $P N$ and GPP, and PN and PD and GPP were -0.10, -0.03 and 0.97, respectively. In the study population characteristics GPP and PD have a high response to selection, unlike the PN. The GPP and PN characteristics are enough to make a selection index, it is advisable to monitor the $P N$ in the initial phase of the program.
\end{abstract}

Keywords: heritability, animal breeding, sheep industry, genetic

Recebido em 14 de junho de 2013

Aceito em 24 de fevereiro de 2015

E-mail: micheleportopires@gmail.com 


\section{INTRODUÇÃO}

No Brasil, a ovinocultura vem crescendo significativamente, o rebanho ovino apresentou crescimento de $21,02 \%$ entre os anos de 2002 a 2013 (IBGE, 2014). O rebanho brasileiro é, em sua maioria, composto por ovinos nativos criados de forma extensiva com pouca ou nenhuma tecnologia empregada; sendo assim, os animais produzidos apresentam baixos índices produtivos. Os animais enviados para os frigoríficos possuem baixa qualidade com idade avançada e mal terminados (SEBRAE, 2005). Dessa forma, as indústrias brasileiras enfrentam dificuldades em atender a demanda e a qualidade exigida pelo mercado consumidor interno, recorrendo à importação de carne ovina.

Entretanto, a ovinocultura brasileira, segundo Costa (2007), vem sofrendo alterações, passando de uma atividade de subsistência para uma atividade empresarial e especializada. Essas mudanças podem ser observadas através da expansão da raça nativa Santa Inês por todo o território brasileiro e pela condução de programas de melhoramento genético focados na seleção dessa raça. Todavia, para que a ovinocultura brasileira se torne competitiva a curto e médio prazo, faz-se necessário o uso do cruzamento com raças especializadas na produção de carne.

A raça Suffolk é uma alternativa para o cruzamento com a raça Santa Inês, pois, além de ser especializada para o corte, também apresenta rusticidade (Alberti Filho et al., 2009). Segundo Fernandes et al. (2007), estudando o desempenho de cordeiros mestiços e puros Santa Inês e Suffolk, concluíram que o desempenho de cordeiros mestiços Santa Inês e Suffolk assemelhou-se ao desempenho de cordeiros puros Suffolk, específicos para corte.

No entanto, para que os efeitos do cruzamento sejam explorados de forma adequada e eficiente, a seleção das raças especializadas para corte deve ocorrer no país. Assim, os parâmetros genéticos desses rebanhos devem ser conhecidos e estudados, viabilizando a formulação de estratégias de seleção.

A literatura brasileira traz escassas referências sobre a estrutura genética da população de Suffolk no país. Para peso ao nascer, ganho de peso do nascimento ao desmame e peso ao desmame, as herdabilidades estimadas variam de 0,06 a 0,09 (Alberti Filho et al., 2009; Alberti Filho et al., 2010; Grion et al., 2010). Existem também alguns trabalhos sobre estimativa de componentes genéticos para características reprodutivas e de escores visuais para a raça (Grion et al., 2009; Cosmo et al., 2010; Grion et al., 2010; Somavilla et al., 2010), mas estes também são escassos.

Dessa forma, o objetivo do trabalho foi estimar os parâmetros genéticos das características peso ao nascer (PN), ganho de peso do nascimento ao desmame (GPP) e peso ao desmame (PD) da raça Suffolk, a fim de fornecer subsídio para a definição de estratégias de seleção para programas de melhoramento genético.

\section{MATERIAL E MÉTODOS}

Os dados analisados, coletados entre os anos de 2007 à 2009, foram referentes a ovinos da raça Suffolk oriundos de uma propriedade localizada no Estado de São Paulo, Brasil, participante do programa de melhoramento genético Ovigol, conduzido pela empresa Aries Reprodução e Melhoramento Genético Ovino Ltda., Brasil em parceria com a empresa AbacusBio Limited, Nova Zelândia.

As características avaliadas foram: peso ao nascer $(\mathrm{PN})$, ganho de peso do nascimento ao desmame (GPP) e peso ao desmame (PD). Para a definição dos efeitos não genéticos que foram incluídos no modelo misto, utilizou-se o procedimento GLM do programa computacional SAS (Statistical Analysis System). O modelo é descrito abaixo:

$Y=X \cdot b+Z \cdot a+W \cdot e p_{m}+e$

Em que $Y=$ vetor de observações para uma específica característica no animal; $b=$ vetor de efeitos fixos; $a=$ vetor de efeitos genéticos aditivos; $e p_{m}=$ vetor de efeitos de ambiente permanente materno; $X, Z$ e $W=$ matrizes de incidências para os efeitos fixos, genéticos aditivos e de ambiente permanente materno, respectivamente e $e=$ vetor de efeitos aleatórios residuais associados às observações.

Os efeitos fixos foram compostos por sexo (dois 
níveis), idade como covariável de efeito quadrático (média de idade ao desmame de 90 dias) e grupo de contemporâneos. $\mathrm{O}$ grupo de contemporâneos foi constituído por animais nascidos no mesmo ano e pelo mesmo tipo de parto (simples, duplo ou triplo), totalizando nove grupos de contemporâneos.

As pressuposições assumidas em relação aos componentes foram:

$$
\begin{aligned}
& E[y]=X \cdot \beta \\
& E\left[\begin{array}{l}
a \\
p \\
e
\end{array}\right]=\left[\begin{array}{l}
0 \\
0 \\
0
\end{array}\right] \cdot \operatorname{var}\left[\begin{array}{l}
a \\
p \\
e
\end{array}\right]=\left[\begin{array}{lll}
A \sigma_{a}^{2} & 0 & 0 \\
0 & I \sigma_{p}^{2} & 0 \\
0 & 0 & I \sigma_{e}^{2}
\end{array}\right]
\end{aligned}
$$

Em que: $A=$ matriz de parentesco; $\sigma_{a}^{2}=$ componente de variância genética aditiva; $\sigma_{p}^{2}=$ componente de variância de efeito permanente materno; $\sigma_{e}^{2}=$ componente de variância residual e $I=$ matriz identidade.

Após a consistência dos dados, restaram 1.039, 636 e 649 observações para PN, GPP e PD, respectivamente. Para a realização da consistência e recodificação do pedigree, foi utilizado o programa computacional Relax2: pedigree analysis program (Strandén e Vouri, 2006) e, para as estimativas dos componentes de (co)variâncias e dos parâmetros genéticos, o programa computacional utilizado foi o WOMBAT (Meyer, 2006), utilizando-se o algoritmo $A I-R E M L$ e critério de convergência $10^{-8}$, empregando modelo animal em análise multicaracterísticas. $\mathrm{O}$ efeito genético materno não foi incluído no modelo devido à ausência de informações necessárias; entretanto, conhecendose a importância dos efeitos maternos sobre as características de crescimento dos cordeiros, incluíram-se os efeitos de ambiente permanente materno, a fim de melhorar a qualidade das estimativas (Lewis e Brotherstone, 2002; Matika et al., 2003; Sarmento et al., 2006; Abbassi et al., 2006; Mandal et al., 2006).

\section{RESULTADOS E DISCUSSÃO}

Os resultados apresentados nas Tabelas 1 e 2 foram obtidos de dados de desempenho de ovinos da raça Suffolk e apresentam a estatística descritiva e os componentes de variância e parâmetros genéticos para as características estudadas, respectivamente.

A baixa herdabilidade para PN nessa população pode ser devido a dois fatores. $\mathrm{O}$ primeiro referese à alta variância ambiental em comparação aos demais componentes. Geralmente, a mensuração da característica PN nas propriedades brasileiras é realizada com balanças de baixa precisão e com intervalos de mensuração diferentes (logo após o parto ou até 48 horas após o nascimento). Outro fator que contribui para tal variância diz respeito ao efeito de ambiente permanente materno, que contribuiu com $21,51 \%$ da variação fenotípica total $\left(\boldsymbol{\sigma}_{\mathrm{p}}^{\mathbf{2}}\right)$, indicando significativa influência dos efeitos maternos sobre o peso ao nascer. Maniatis e Pollot (2002) estimaram a contribuição da variância do efeito de ambiente permanente materno sobre a variância fenotípica do PN de $32 \%$ em um rebanho fechado de ovinos Suffolk.

Tabela 1. Médias, número de observações, desvios padrão e coeficiente de variação das características de crescimento de ovinos da raça Suffolk após análises de consistência

\begin{tabular}{ccccc}
\hline Características & $\begin{array}{c}\text { Número de } \\
\text { observações }\end{array}$ & $\begin{array}{c}\text { Média } \\
(\mathrm{kg})\end{array}$ & $\begin{array}{c}\text { Desvio padrão }(\mathrm{kg}) \\
\text { PN }\end{array}$ Coeficiente de $^{\text {variação }(\%)}$ \\
\hline GPP & 636 & 4,22 & 1,00 & 23,71 \\
PD & 649 & 0,26 & 0,08 & 32,75 \\
\hline
\end{tabular}

PN $=$ Peso ao nascer; GPP $=$ Ganho de peso do nascimento ao desmame e PD = Peso ao desmame (média de idade de 90 dias). 
Tabela 2. Estimativas das herdabilidades, com respectivos erros padrão (em parênteses), variâncias genéticas aditivas, variâncias de efeito permanente materno e as variâncias residuais pelo modelo multicaracterísticas para ovinos da raça Suffolk

\begin{tabular}{cccccc}
\hline Características & $\mathrm{h}^{2}$ & $\sigma_{\mathrm{a}}^{2}$ & $\sigma_{\mathrm{apm}}^{2}$ & $\sigma_{\mathrm{e}}^{2}$ & $\mathrm{c}^{2}$ \\
\hline PN & $0,06( \pm 0,06)$ & 0,0484 & 0,16481 & 0,55289 & 0,2151 \\
GPP & $0,42( \pm 0,16)$ & 0,0017 & 0,00017 & 0,00227 & 0,0413 \\
PD & $0,37( \pm 0,15)$ & 12,249 & 1,8673 & 18,763 & 0,0568 \\
\hline
\end{tabular}

$\mathrm{PN}=$ Peso ao nascer; $\mathrm{GPP}=$ Ganho de peso médio diário do nascimento ao desmame; $\mathrm{PD}=$ Peso ao desmame (média de idade de 90 dias); $h^{2}=$ Herdabilidade; $\sigma_{a}^{2}=$ Variância genética aditiva; $\sigma_{\text {apm }}^{2}=$ Variância de efeito ambiental permanente materno; $\sigma_{\mathrm{e}}^{2}=$ Variância ambiental ou residual e $\mathrm{c}^{2}=$ Proporção de variância devido ao efeito de ambiente permanente materno.

O segundo fator que poderia explicar tal magnitude pode estar relacionado com os efeitos da seleção (natural ou artificial) sobre a raça ao longo dos anos, reduzindo sua variância genética aditiva para essa característica. Vários trabalhos realizados com a raça Suffolk e mestiço Suffolk obtiveram herdabilidades variando de 0,05 a 0,19 para PN (Simm et al., 2002; Maniatis e Pollot, 2002; Maxa et al., 2007; Alberti Filho et al., 2009; Tamioso et al., 2013; Tamioso et al., 2014).

Herdabilidades moderadas foram estimadas para GPP e PD, cujos valores foram superiores aos encontrados na literatura para animais puros e mestiços Suffolk, os quais variaram de 0,07 a 0,30 (Sherestha et al., 1986; Maxa et al., 2007; Alberti Filho et al., 2010; Tamioso et al., 2013) para GPP e de 0,07 a 0,26 (Notter, 1998; Simm et al., 2002; Komlósi, 2008; Grion et al., 2010; Tamioso et al., 2013; Tamioso et al., 2014) para PD.

As herdabilidades estimadas para GPP e PD podem estar relacionadas com o manejo adotado pela propriedade, a qual faz uso do creepfeeding. $\mathrm{O}$ creep-feeding proporcionou as mesmas condições de alimentação para todos os animais, diminuindo, assim, as variâncias ambientais e permitindo a expressão do potencial genético dos mesmos, possibilitando a observação de cordeiros com diferentes potenciais genéticos.

A Tabela 3 apresenta as correlações entre as características estudadas para ovinos da raça Suffolk.

Tabela 3. Estimativas das correlações genéticas (acima da diagonal), erros padrão (entre parênteses) e correlações fenotípicas (abaixo da diagonal) entre as características de crescimento para ovinos da raça Suffolk

\begin{tabular}{cccc}
\hline Características & PN & GPP & PD \\
\hline PN & & $-0,1006$ & $-0,0283$ \\
& - & $( \pm 0,48)$ & $( \pm 0,49)$ \\
GPP & 0,117 & - & 0,9677 \\
& $( \pm 0,04)$ & - & $( \pm 0,02)$ \\
PD & 0,274 & 0,9557 & \\
\hline
\end{tabular}

$\mathrm{PN}=$ Peso ao nascer; GPP $=$ Ganho de peso ao pré-desmame e $\mathrm{PD}=$ Peso ao desmame (média de idade de 90 dias).

As correlações genéticas entre PN e GPP e entre PN e PD estimadas foram próximas a zero. Tais correlações indicam que a seleção aplicada a essa população em uma das características (GPP ou PD) não levará a resposta indireta para $\mathrm{PN}$, resultado favorável à aplicação de estratégias que visam elevar o desempenho das características GPP e PD sem elevar o PN. Todavia, os erros padrão para as estimativas das correlações foram altos, indicando elevado risco na tomada de estratégias de seleção que envolva o PN e GPP ou PD. Para minimizar os erros padrão das 
correlações e diminuir o risco na construção de um índice composto pelas características citadas anteriormente, seria necessário coletar mais informações sobre GPP e PD.

De acordo com o exposto acima, a ponderação para PN em um índice de seleção composto por PN e GPP ou PD dependerá do PN do rebanho. Se este for baixo a ponto de prejudicar a viabilidade do cordeiro, então se deve ponderá-lo com valor positivo e com magnitude que permita selecionar reprodutores ou matrizes que incrementem o PN. Caso esteja adequado, a ponderação deve ser mínima; por outro lado, se o PN estiver causando partos distócicos, então a ponderação deve ser negativa e com magnitude que permita selecionar reprodutores ou matrizes que diminuam o PN das gerações seguintes.

A correlação predita entre GPP e PD foi de magnitude alta e positiva, indicando que a seleção para uma das características levará ao progresso genético da outra. Para a adoção de estratégias de seleção em programas de melhoramento genético, a magnitude da correlação estimada se apresenta favorável, uma vez que se pode trabalhar com ambas ou uma das características em um índice de seleção. A aplicação conjunta do ganho de peso ao prédesmame e do peso ao desmame em um índice de seleção somente se justificaria se as correlações existentes com outras características fossem antagônicas.

\section{CONCLUSÃO}

Para a população estudada, a melhor estratégia de seleção a ser adotada é o uso da característica GPP que, por apresentar maior herdabilidade, possibilitará maior resposta à seleção em relação ao PD.

As características GPP e PD apresentam herdabilidades moderadas e, portanto, produzirão maior resposta à seleção, o que não ocorrerá para o PN. As características PN e GPP são suficientes para compor um índice de seleção, em que a ponderação para PN será relativamente menor que aquela associada à GPP.

\section{AGRADECIMENTOS}

Ao Msc. Bruno Fernandes Salles, pelo banco de dados concedido, e aos amigos de trabalho do LuCCA-Z, pela ajuda no desenvolvimento do mesmo.

\section{REFERÊNCIAS}

ABBASSI, M.A; VAEZ TORSHIZI, R; NEJATI JAVAREMI, A; OSFOORI, R. Introducing the suitable model for analysis of the lamb body weight using log likelihood ratio. In: ANUAL MEETING EUROPEAN FEDERATION OF ANIMAL SCIENCE, 57., 2006, Antalya, Turkey. Abstracts... Antalya: Wageningen Academic Publishers, 2006. p.18-23. Disponível em: $<\mathrm{http} / / /$ PDw.eaap.org/Previous_Annual_Meetings/200 6Antalya/sessions/session $03 \cdot \overline{\mathrm{ht}} \mathrm{\textrm {t }}>$. Acessado em: 20 mar. 2011.

ALBERTI FILHO, J.L.; DIAS, L.T.; TEIXEIRA, R.A. Comparação de diferentes modelos para a estimação de herdabilidade para peso, em diferentes idades, em ovinos. In: REUNIÃO ANUAL DA SOCIEDADE BRASILEIRA DE ZOOTECNIA, 46., 2009, Maringá. Anais ... Maringá: Sociedade Brasileira de Zootecnia, 2009.

ALBERTI FILHO, J.L.; DIAS, L.T.; TEIXEIRA, R.A. Influência de efeitos maternos na estimação de herdabilidade para o ganho de peso no período pré e pós-desmama em ovinos Suffolk. In: SIMPÓSIO BRASILEIRO DE MELHORAMENTO ANIMAL, 8., 2010, Maringá. Anais... Maringá: [s.n.], 2010. Disponível em: <http://sbmaonline.org.br/anais/ viii/trabalhos/>. Acessado em: 20 mar. 2011.

COSMO, T.R.; DIAS, L.T.; TEIXEIRA, R.A. Parâmetros genéticos para peso corporal e escores visuais após a desmama em cordeiros Suffolk. In: SIMPÓSIO BRASILEIRO DE MELHORAMENTO ANIMAL, 8., 2010, Maringá. Anais... Maringá: [s.n.], 2010. Disponível em: <sbmaonline.org.br/anais/viii/ trabalhos/pdfs/C510.pd>. Acessado em: 19 mar. 2011.

COSTA, N.G. A cadeia produtiva de carne ovina no Brasil rumo às novas formas de organização da produção. 2007. 182f. Dissertação (Mestrado em Agronegócios) - Faculdade de Agronomia e Medicina Veterinária, Universidade de Brasília, Brasília, DF.

FERNANDES, M.A.M; MONTEIRO, A.L.G; BARROS C.S. et al. Desempenho de cordeiros puros e cruzados Suffolk e Santa Inês. Rev. FZVA, v.14, p.207216, 2007.

GRION, A.L; DIAS, L.T.; TEIXEIRA, R.A. Estimativas de parâmetros genéticos para perímetro escrotal e intervalo entre partos em ovinos Suffolk. In: SIMPÓSIO PARANAENSE DE OVINOCULTURA, 14.; SIMPÓSIO PARANAENSE DE CAPRINOCULTURA, 2.; SIMPÓSIO SUL BRASILEIRO DE OVINOS E CAPRINOS, 2., Curitiba. Anais... Curitiba: LAPOC-UFPR, 2009. 
GRION, A.L.; DIAS, L.T.; TEIXEIRA, R.A. Estimativas de parâmetros genéticos para características reprodutivas e de crescimento em ovinos Suffolk. In: SIMPÓSIO BRASILEIRO DE MELHORAMENTO ANIMAL, 8., 2010, Maringá. Anais... Maringá: [s.n.], 2010. Disponível em: $<$ sbmaonline.org.br/anais/viii/trabalhos/pdfs/C505.pdf >. Acessado em: 22 mar. 2011.

PRODUÇÃO da pecuária municipal - 2013. Instituto Brasileiro de Geografia e Estatística, 2014. Disponível em:

$<$ http://www.ibge.gov.br/home/estatistica/economia/pp m/2013/default.shtm>. Acessado em: 26 jan. 2015.

KOMLÓSI, I. Genetic parameters for growth traits of the Hungarian Merino and meat sheep breeds in Hungary. Appl. Ecol. Environm. Res. v.6, p.77-84, 2008.

LEWIS, R.M.; BROTHERSTONE, S. A genetic evaluation of growth in sheep using random regression techniques. Anim. Sci., v.74, p.63-70, 2002.

MANDAL, A.; NESER, F.W.C.; ROUT, P.K. et al. Estimation of direct and maternal (co)variance components for pre-weaning growth traits in Muzaffarnagari sheep. Livest. Sci., v.99, p.79-89, 2006.

MATIKA, O.; VAN WYK, J.B.; ERASMUS, G.J.; BAKER, R.L. Genetic parameter estimates in Sabi sheep. Livest. Prod. Sci., v.79, p.17-28, 2003.

MAXA, J.; NORBERG, E.; BERG, P.; PEDERSEN, J. Genetic parameters for growth traits and litter size in Danish Texel, Shropshire, Oxford Down and Suffolk. Small Rumin. Res., v.68, p.312-317, 2007.

MANIATIS, N.; POLLOTT, G.E. Maternal effects on weight and ultrasonically measured traits of lambs in a small closed Suffolk flock. Small Rumin. Res., v.45, p.235-246, 2002

MEYER, K. Wombat: a program for mixed model analyses by restricted maximum likelihood. Armidale, Australia University of New England, 2006. 66p.
NOTTER, D. R. Genetic parameters for growth traits in Suffolk and Polypay sheep. Livest. Prod. Sci., v.55, p.205-213, 1998.

SARMENTO, J.L.R.; TORRES, R.A.; SOUSA, W.H. et al. Estimação de parâmetros genéticos para características de crescimento de ovinos Santa Inês utilizando modelos uni e multi-característica. Arq. Bras. Med. Vet. Zootec., v.58, p.581-589, 2006.

INFORMAÇÕES de mercado sobre caprinos e ovinos. Brasília, DF: Sebrae, 2005. 73 p.

SHERESTHA, J.N.B.; VESELY, J.A.; CHESNAIS, J.P. Genetic and phenotypic parameters for daily gain and body weights in Suffolk lambs. Can. J. Anim. Sci. v.65, p.575-582, 1986.

SIMM, G.; LEWIS, R.M.; GRUNDY, B.; DINGWALL, W.S. Responses to selection for lean growth in sheep. Anim. Sci., v.74, p.39-50, 2002.

SOMAVILLA, A.L.; DIAS, L.T.; TEIXEIRA, R.A. Estimativas de parâmetros genéticos para conformação, precocidade e musculatura à desmama em ovinos Suffolk. In: SIMPÓSIO BRASILEIRO DE MELHORAMENTO ANIMAL, 8., 2010, Maringá. Anais... Maringá: [s.n.], 2010. Disponível em: <sbmaonline.org.br/anais/viii/trabalhos/pdfs/C502.pd> . Acessado em: 19 mar. 2011.

STRANDÉN, I.; VUORI, K. RelaX2: pedigree analysis program. In: WORLD CONGRESS ON GENETICS APPLIED TO LIVESTOCK PRODUCTION, 8., 2006, Belo Horizonte. Proceedings... Belo Horizonte: [s.n.], 2006. p.27-30.

TAMIOSO, P.R.; ALBERTI FILHO, J.L.; DIAS, L.T.; TEIXEIRA, R.A. Estimates of (co)variance components and genetic parameters for growth traits in Suffolk lambs. Cienc. Rural, v.43, p.2013.

TAMIOSO, P.R.; DIAS, L.T.; TEIXEIRA, R.A.; SILVA, C.J.A. Estimativas de parâmetros genéticos para características de crescimento de cordeiros mestiços Suffolk. Cienc. Anim. Bras., v.15, p.414-419, 2014. 